\title{
DRG statt Diabetologie
}
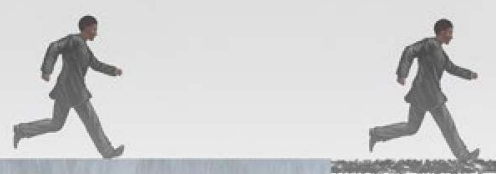

s.

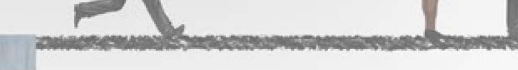

Das Honorierungssystem „Disease Related Grouping“ (DRG) wurde in Deutschland vor Jahren als Steuerungselement eingeführt, um die steigenden Gesundheitsausgaben im stationären Sektor einzudämmen. Zwar ist es gelungen, die Liegezeiten in Kliniken deutlich zu senken, jedoch hat dieses Abrechnungssystem die Kostensteigerung im stationären Sektor nicht wesentlich bremsen können. Manche Kritiker meinen, dass durch das DRG-System bestimmte „lukrative Operationen“ häufiger geworden sind. Zusätzlich wurde der Begriff der „blutigen Entlassung " von Hausärzten geprägt, die sich mit Patienten konfrontiert sehen, die nach kurzen Krankenhausaufenthalten noch intensive medizinische Hilfe benötigen.

Die Einführung der DRGs in Deutschland hat jedoch weitere Auswirkungen auf ganze Fachgebiete, wie man am Beispiel der Diabetologie darstellen kann. Bedingt durch die Vergütung im DRG-System und die strengen Vorgaben der Krankenkassen, wann stationäre Aufenthalte bei Diabetes mellitus den Krankenhäusern vergütet werden, wurde die stationäre Diabetologie auf die Therapie bei schwerem diabetischem Fußsyndrom reduziert. Klassische Diabeteskliniken oder Krankenhausabteilungen wurden vielerorts - oder werden aktuell - geschlossen. Ansonsten verirren sich Patienten mit der Hauptdiagnose „Diabetes mellitus“ nur noch im Fall von Notfällen wie schweren Hypoglykämien oder Ketoazidosen in Krankenhäuser. Alles andere wird durch diabetologische Schwerpunktpraxen abgedeckt, von denen es in Deutschland laut Deutscher Diabetesgesellschaft (DDG) ca. 800 gibt.

\section{Diabetologisches Wissen geht verloren}

Durch diese zielstrebig umgesetzte Ökonomisierung in der Medizin ist jedoch das Wissen zum Thema „Diabetes mellitus“ in den Krankenhäusern so nach und nach verloren gegangen. Wenn überhaupt kümmern sich in vielen Kliniken engagierte Krankenschwestern um erhöhte Blutzuckerwerte bei stationären Patienten. Besonders Patienten mit Typ-1-Diabetes können bei stationären Aufenthalten aufgrund anderer Erkrankungen viele erschreckende Geschichten erzählen. Das vor wenigen Jahren durch die DDG eingeführte Zertifikat des „diabetesgeeigneten Krankenhauses“ wurde bisher gerade mal 70 Kliniken in Deutschland zuerkannt. Stationäre Abteilungen in Krankenhäusern sind aber die wesentlichen Institutionen, in denen die Ausbildung junger Kolleginnen und Kollegen in Deutschland stattfindet. Die logische Konsequenz aus der zuvor dargestellten Entwicklung des Niedergangs der stationären Diabetologie ist, dass künftige Generationen von Ärzten kaum noch Wissen haben werden, wie man Patienten mit der Volkserkrankung Diabetes mellitus behandeln kann. Diese Entwicklung lässt sich sehr gut an der Menge der Weiterbildungsprüfungen der Ärztekammer nachweisen. So wurden in den letzten 5 Jahren jährlich nur 100 Weiterbildungsprüfungen für die Weiterbildung Diabetologie abgenommen - bundesweit! Im Bereich der Notfallmedizin wurden im gleichen Zeitraum im Mittel über 2000 und in der Intensivmedizin über 650 Weiterbildungsprüfungen pro Jahr abgelegt. Sogar die Weiterbildung Naturheilkunde lag mit jährlich 120 Prüfungen höher als in der Diabetologie. Nur die Weiterbildung in der Homöopathie mit knapp 60 Prüfungen pro Jahr scheint noch etwas unattraktiver als die Diabetologie zu sein. Die geringe Weiterbildung zum Diabetologen ist aber nur ein Teilaspekt, denn die meisten Patienten werden von Hausärzten betreut. Doch wo werden diese ausgebildet?

\section{Planwirtschaft ohne Plan?}

Die Ökonomisierung in der Medizin hat ihre Vorbilder in der Großindustrie. Dort wurden auch ganze Berufsgruppen wegrationalisiert - jedoch nur dann, wenn Alternativen bestanden. So wichen der Heizer in Dampfloks der Elektrolok und Fließbandarbeiter in der Autoproduktion den Robotern. In der Medizin aber werden Fachbereiche zu Grabe getragen, die nicht nur heute wichtig sind, sondern in der Zukunft noch viel wichtiger werden als sie heute sind!

Da man grundsätzlich das Gute im Menschen sehen sollte, muss man davon ausgehen, dass niemand diese Entwicklung gewollt hat, um vielleicht mehr medizinische Komplikationen für attraktive DRGs zu generieren. Vielmehr war scheinbar die Abteilung „Jugend forscht" am Werk: hoch motiviert, aber leider ohne substanzielles Wissen. Oft hat man bei solchen Entwicklungen den Eindruck, dass die Strukturen des Gesundheitswesens die letzten Residuen des Sozialismus auf deutschen Boden sind, quasi Planwirtschaft, aber ohne Plan.

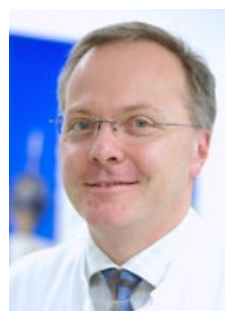

Prof. Dr. med. Stephan Martin

Verbund der Katholischen Kliniken Düsseldorf (VKKD), Chefarzt für Diabetologie und Direktor des Westdeutschen Diabetesund Gesundheitszentrum (WDGZ) Hohensandweg 37, 40591 Düsseldorf Stephan.Martin@vkkd-kliniken.de 